\title{
Intuitive inventory management: An experimental system
}

\author{
JOACHIM MEYER and MOSHE EBEN-CHAIME \\ Ben-Gurion University of the Negev, Beer-Sheva, Israel
}

\begin{abstract}
The way people intuitively manage inventories is both an important issue in its own right and a context for research on various basic theoretical questions. The subject has not yet been systematically studied, owing partially to the lack of appropriate research tools. This paper presents some of the key concepts in formal inventory theory and describes an experimental system for the study of intuitive inventory management. This system is a computer program that allows subjects to interact with a simulated inventory system. Numerous system parameters can be manipulated, including demand functions, prices, costs, and the display of data. The usefulness of the system is demonstrated via the study of the effects of three parameters on intuitive inventory management in an illustrative experiment. Possible future uses of the system in basic and applied research and in training are indicated.
\end{abstract}

Mathematicians, management scientists, and statisticians have developed numerous formal, mathematical tools that permit the rationalization of decision-making processes. Yet despite the increasing use of these tools, most decisions are still based on intuition and heuristics. The study of these intuitive processes has been the subject of many papers. Some have dealt with characteristics of the human decision-making process in general (e.g., the papers in Kahneman, Slovic, \& Tversky, 1982), whereas others have dealt with more specific issues, such as intuitive reasoning about problems in physics or in technological systems (e.g., the papers in Gentner \& Stevens, 1983), the intuitive solving of statistical problems (e.g., Meyer \& Shinar, 1992), or lay theories in the social sciences (Furnham, 1988).

Recently, there have been some attempts to study intuitive behavior in tasks related to operations research. For example, people's behavior in a scheduling task under various conditions of workload has been investigated by Moray, Dessouky, Kijowski, and Adapathya (1991), and the importance of understanding aspects of the human factor in the production management process was stressed by Sanderson (1989). Although most decisions in these fields are made intuitively, few systematic studies exist yet. This paper presents a tool for the study of intuitive decision making in one such domain, that of inventory management-namely, the decisions regarding the timing and quantity of purchasing various goods.

The first author is now at the Research Center for Work Safety and Human Engineering, Technion--Israel Institute of Technology, Haifa, Israel. This study was partially supported by the Center for Ergonomics and Safety, Ben-Gurion University of the Negev, Beer-Sheva, Israel. We gratefully acknowledge the assistance of $A$. and $R$. Matalon, S. Efal, and A. Silverstein in programming the experimental system and conducting initial usability tests. All correspondence concerning this paper should be addressed to M. Eben-Chaime, Department of Industrial Engineering and Management, Ben-Gurion University, 84105 Beer-Sheva, Israel (e-mail: even@bgumail.bgu.ac.il).
Few systematic studies have addressed the question of how people actually manage an inventory system (see, e.g., Kleiter, 1970). However, most decisions (even in commercial settings) concerning the time and quantity of the ordering of various commodities are still made intuitively, since it is difficult and costly to formulate mathematical models for each item and condition. From an applied point of view, the study of intuitive decision-making processes may reveal their characteristics, and especially the possible existence of systematic deviations from the optimal models. These deviations can then be stressed in specific training programs, in which decision makers can learn about these biases and how to avoid them. In addition, the study of these issues provides for the redesign of the displays and interfaces in decision support systems, in order to eliminate possible errors that may result from the intuitive problem-solving methods. Finally, in marketing, being aware of the possible biases in a client's reasoning may allow marketing tactics to be devised that address these biases specifically and thereby increase sales.

From a theoretical point of view, the systematic study of intuitive decisions in inventory management may lead to a better understanding of people's ability to reason about complex domains, where multivariate interactions govern the performance of a system. Inventory management is a typical complex problem-solving situation. Funke (1991) identified six major features that characterize complex problem solving: (1) "intransparency," whereby only some of the variables lend themselves to direct observation, while others have to be inferred indirectly; (2) "polytely," or the existence of multiple and often contradictory goals; (3) the "complexity of the situation," defined by the number of variables, the possibility to control the system, and the dynamic aspects of the system; (4) the "connectivity of the variables," which consists of the degree to which changes in one variable affect the status of many other related variables; (5) "dynamic developments," due to the existence of time pressure, which involve rapid changes and the possi- 
ble deterioration of conditions when inappropriate actions are taken; and (6) the existence of "time delayed effects," causing actions to take effect only after a certain time period has elapsed. All six features are important for determining people's performance with complex systems, and most of them are involved in inventory management tasks.

Often the situation in inventory management is intransparent, in the sense that not all variables, such as the demand patterns for items, are known. There may exist multiple objectives, such as the attempt to minimize inventories as opposed to the attempt to avoid shortages. The complexity of the situation may vary considerably, according to the number of items in stock and the variables affecting their management. The variables are strongly connected, and different (often nonlinear) relations exist between variables. Inventory management is a dynamic process, in which situations change as a function of time. Finally, inventory'management often involves time delays, resulting from delays in the supply of ordered goods. Hence the features of complex problem solving can easily be incorporated in inventory management tasks.

One major advantage of using inventory management as a framework for studying complex decision making is the fact that it is relatively easy to explain the meanings of the variables to participants in experiments, without the need to resort to artificial and unrealistic variables. Most people understand the meaning of the various factors involved in inventory management without difficulty, although they may not be familiar with the complex relations that exist between them. In this respect inventory management has definite advantages as a framework for the study of intuitive decision making, as opposed to other domains that have been used in previous studies that often require extensive training of the subjects in order to provide them with some basic understanding of the meaning of the variables in the system. (For a review of the research on complex problem solving, see Funke, 1991.)

The use of the intuitive inventory management domain allows the design of complex experiments in which people's ability to understand various patterns of interaction between variables may be studied. It is also possible to gain some insight into the systematic changes in patterns of behavior that occur when subjects gather experience in working with a specific system, and into the way in which they learn to deal with the complex interactions that exist between the different variables. In this paper, an experimental system is presented that permits the systematic study of performance in numerous inventory management situations. To facilitate the presentation of the system, a brief description of inventory systems and their management will be given first. (For a more detailed discussion, any one of the many textbooks on the subject may be consulted--e.g., Hax \& Candea, 1984; Johnson \& Montgomery, 1974.)

\section{INVENTORY SYSTEMS AND MANAGEMENT}

In many cases, goods flow through systems. An input process governs the entrance, along time, of a commod- ity into the system; an output process dictates how this commodity exits the system. When the amount entering the system exceeds the amount leaving the system, the surplus is stored and an inventory is created. The output is a response to another process, the demand process. When the demand exceeds the input plus the amount in stock (if there is any), a shortage occurs. Some of this extra demand may be back logged, or back ordered, creating a negative inventory.

Inventories can be constructed accidentally because of errors, or they may be imposed by technological constraints. Our interest, however, is in deliberately constructed inventories. Several factors can justify inventory construction, most of which are economic. Among them are decreased unit costs, due to economy of scale, and the chance to take advantage of future price increases. Inventories also serve as safeguards against demand fluctuations and as means for reducing the dependency between successive production stages which might otherwise lead to frequent material shortages and shutdowns. Finally, inventories protect against losses resulting from shortages due to the combined effect of uncertainties in the demand and the length of the lead times - that is, the time span between the issuing of an order and its delivery.

Inventory management requires the careful balancing of the various benefits with the costs associated with the existence of inventories. The relevant costs may be grouped into four categories: (1) fixed procurement costs, or the costs that are unaffected by procurement quantities; (2) variable procurement costs; (3) shortage costs; and (4) holding, or carrying, costs. Fixed costs per unit decrease as the ordered quantity increases, which motivates large order quantities. Large order quantities also become attractive when quantity discounts are offered by the supplier. Finally, large order quantities result in higher inventory levels, which reduce shortages and the associated costs. On the other hand, holding costs, which increase with inventory levels, motivate the frequent ordering of small quantities. The inventory manager has to determine the optimal tradeoff between the conflicting considerations.

The simplest model may serve as an illustration. It is a single-item deterministic model (i.e., no uncertainty exists), in which shortages are not allowed and deliveries are made instantaneously. System behavior over time is shown in Figure 1. The vertical segments represent elevations of inventory levels due to deliveries, and the diagonal segments represent consumption according to a constant demand rate of $D$ units per unit of time (usually a year). The total costs (TC) as a function of the order quantity, $Q$, the model's decision variable, consists of three components, as follows:

$$
\mathrm{TC}(Q)=C D+A D / Q+h Q / 2 .
$$

The first component accounts for the variable procurement costs at a fixed price of $C \$ /$ unit. Since no discounts are applied, this term is independent of $Q$. The second component accounts for fixed procurement costs of $A \$ /$ order, where $D / Q$ equals the number of orders issued during a time unit. The last term accounts for the hold- 


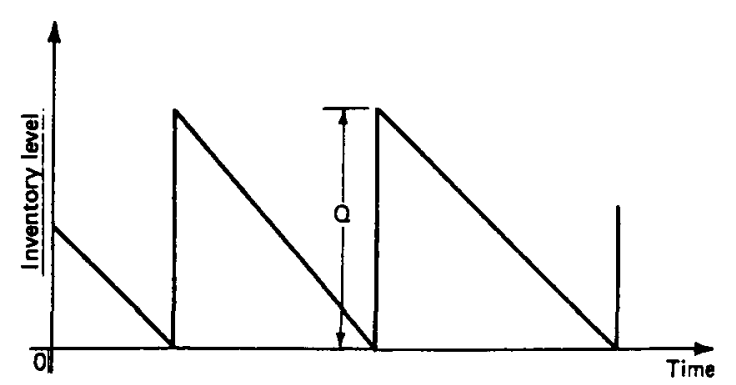

Figure 1. The simple single-item inventory model.

ing costs of $h \$$ per unit per time unit, where $Q / 2$ is the average inventory level throughout the time period. The cycle length - the time between two consecutive deliveries - is given in $Q / D$ time units. The reorder point is the inventory level, $r$, which indicates that an order should be placed and is given in terms of the lead time, $\tau$, and the number of orders outstanding at the time when the order is placed, $m=\lfloor\tau D / Q\rfloor$, or the greatest integer less than or equal to $\tau D / Q$ :

$$
r=\tau D-m Q .
$$

The optimal quantity, $Q^{*}$, which minimizes TC, is given by the classic economic order quantity (EOQ) formula:

$$
Q^{*}=\sqrt{2 A D / h}
$$

Substituting $Q^{*}$ in TC, the minimum cost per unit of time (usually, annual cost) is given in terms of the model parameters:

$$
\mathrm{TC}\left(Q^{*}\right)=C D+\sqrt{2 A D h} .
$$

For example, suppose the annual demand for an item is of 2,500 units; it can be purchased at $\$ 25$ per unit, plus $\$ 5$ per order, and the holding costs per unit are $10 \%$ of the purchasing costs annually. The EOQ equals $[2 * 5 * 2,500 / .1 * 25]^{1 / 2}=100$ units per order, or 25 orders per year. The associated minimum annual operation costs are of: $25 * 2,500+[2 * 5 * 2,500 * 2.5]^{1 / 2}=\$ 62,750$. Suppose, also, that the lead time is 1 month, $\tau=1 / 12$. Then, $m=\lfloor 2,500 /(12 * 100)\rfloor=2$, and the reorder point, $r=2,500 / 12-2 * 100=81 / 3$. Thus, when the inventory reaches the level of $81 / 3$ units, an order should be placed to avoid shortages.

One can appreciate the complexity of the decision process even in this very simple case. Imagine the cumulative effect of a multi-item inventory system coupled with constraints (e.g., storage space) under uncertainty, which implies unavoidable shortages. Thus, it is of great interest to learn how human managers deal with this complex task which is often still performed without the use of formal tools.

\section{DESCRIPTION OF THE SOFTWARE}

The IIMS (Intuitive Inventory Management System) is a software package, written in Turbo-Pascal for IBM PCs and compatibles with color graphics. It requires at least $512 \mathrm{~K}$ of RAM and may be run from a hard disk or a floppy disk drive, under MS-DOS 3 or later releases. The software can be obtained by anonymous file transfer protocol (FTP) from the COMPsych archive.

The system simulates the behavior of an inventory system, stocking up to seven items under different conditions, in terms of demand, prices, costs, and supply constraints. This allows the investigator to design and run complex experiments in order to study intuitive inventory management. Subjects view a schematic display of the status of an inventory system showing the inventory level of each item and corresponding parameters. An example of the display is shown in Figure 2.

The display changes dynamically, reflecting fluctuations in the inventory levels as the result of sales and/or purchases. The system operates on a condensed time scale, where intervals at the simulation level represent real time intervals according to a prespecified ratio. For example, $5 \mathrm{sec}$ in the simulation may represent a day in reality. The display would then be updated every $5 \mathrm{sec}$, showing the inventory balances at the end of the day, summarizing daily activities. In addition, accounting information is shown, regarding the costs and revenues from the system. This information is also updated periodically. The subjects' task is to manage the inventory system by deciding when to order and how much to order of each item. One's goal is to maximize the net profit, which is also displayed and updated after each time period.

Operational data are collected and stored by the system, including the periodic demand and sales, as well as the timing and quantity of orders made by the user. The information is stored in the system's log, which may be accessed, allowing the extraction of data relevant for describing the user's activities, and comparing the results with formal inventory models.

The system consists of two main modules: the setup module, in which the parameters for the simulation are specified; and the experimental module, in which the actual experiment is conducted and data on users' activities are collected.

\section{Parameter Setup}

The system is highly flexible regarding the parameters of the experimental run. These can be divided into two major groups: parameters that are related to the inventory system itself, and parameters that govern the experimental run.

The parameters related to the inventory system belong to four categories: demand, prices, costs, and supply constraints. Some of these parameters are common to all items stored in a system; others have different values for each item.

Concerning the demand, the system allows the choice among six different demand functions:

1. Constant demand, where the average demand remains constant over time.

2. Demand with a linear trend, where the average demand increases or decreases linearly over time.

3. Demand with an exponential trend, where the average demand increases or decreases exponentially. 


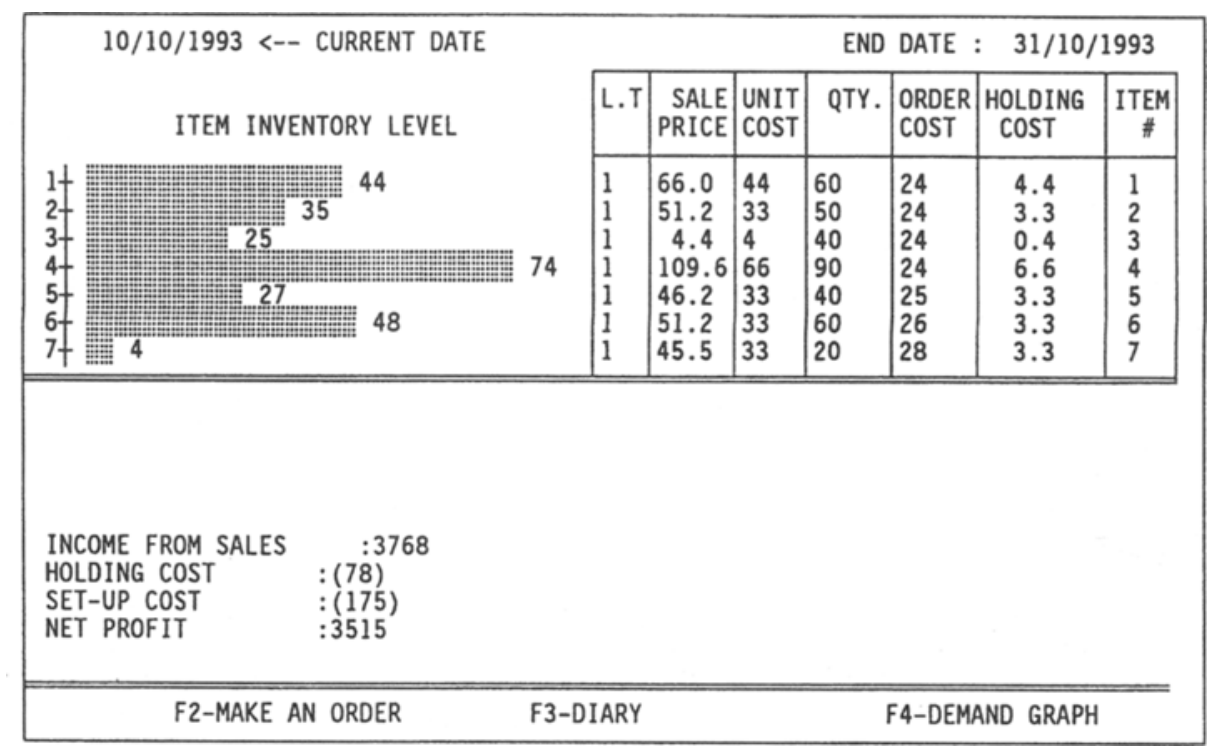

Figure 2. The main screen of the experimental system.

4. Seasonal demand, where seasonal fluctuations may be observed.

5. Seasonal demand with a linear trend, which is a combination of 2 and 4 above.

6. Double seasonal demand where two sinusoidal curves of demand are superimposed, such as when fluctuations occur both monthly and yearly.

For each function, the relevant mathematical parameters have to be specified. Thereby an infinite number of possible demand patterns may be generated. In addition, random noise may be added to the mean demand function, creating normally distributed random deviations from the average pattern. Randomness is determined in terms of the coefficient of variation, which is the ratio of the standard deviation to the mean (i.e., the value calculated by the demand function). Thus, the magnitude of the random noise depends on the mean demand value. Either a single demand function, common to all items, or several functions, one for each item, may be specified. It is also possible to use a preconstructed file with demand values instead of using a demand function. Exact demand patterns can thereby be used repeatedly.

The item sale prices constitute the second parameter category. They are separately specified for each item and remain constant during an experimental run.

The third category includes cost parameters, such as purchasing costs, inventory holding costs, and ordering costs. Purchasing costs per unit are separately defined for each item. Inventory holding costs are the sum of the cost associated with the invested capital and the maintenance cost. Capital costs are the product of the price of the money (e.g., interest rate) and the unit purchasing cost. Both the interest rate and the maintenance cost per unit are specified globally for a given experimental run. The ordering costs contain a global parameter of cost per order (i.e., the cost for making an order, no matter which and how many items are ordered), and specific ordering costs, differing for the different items.

The supply constraint parameters are the maximum quantity that may be ordered for each item in a single order, and the lead time (i.e., the time between placing an order and receiving the merchandise). Both parameters are defined separately for each item.

For each experimental run, a number of parameters have to be provided. First, the number of items stored in the inventory system (from 1 to 7 ) has to be specified. Next, the time frame and scale have to be defined. The time frame is the duration of the experiment in minutes, while the scale is the corresponding duration, in days, of the inventory system. For example, specifying a frame of 5 min may allow a scale of 30 days, each lasting $10 \mathrm{sec}$, or a scale of 60 days, where each day lasts for $5 \mathrm{sec}$. In addition, the rate of information update is defined in terms of days in the inventory system. The displays showing the current state of the inventory and the accounting information are updated either daily, or at a lower frequency, chosen by the experimenter.

Finally, two display options have to be defined. One is the scale of the graphic display of the inventory levels for the different items. These are horizontal bar graphs, where the length of the bar represents the number of units in stock of each item (see Figure 2). The scale is either fixed, with the largest maximum order quantity as the maximum value, or it is dynamic, with the maximum value that of the current largest inventory level among all items.

The second display option concerns the possibility of viewing a vertical bar graph, showing the daily demand. Subjects may, when given the possibility of viewing the graph, interrupt the experimental run at any time by pushing the F4 key, specifying which item's demand graph they would like to see. This leads to the display of the appropriate graph (see Figure 3) until the subjects press the Enter key. 


\section{Experimental Module}

During the experiment, the subject sees the display shown in Figure 2. The top line presents the current date and the experiment's termination date on the experimental calendar. Below, on the right side of the screen, appears a table of the basic information regarding the different items. For each item, the lead time (in days), sale price, purchasing cost, maximum order quantity, ordering cost, and annual holding cost per unit are given. On the left-hand side of the screen, the current inventory levels of all items are shown in a horizontal bar chart format. The exact numeric level is also specified.

The bottom of the screen contains financial information about the system, including: (1) the income from sales, (2) the inventory holding costs, (3) the ordering costs, and (4) the net profit, which equals the income less the inventory holding costs and the ordering costs. This information summarizes the financial results of the system from the beginning of the experiment up to the current date. Participants are instructed to try to maximize the net profit. It is possible to provide cash incentives, by paying for participating in the experiment according to the net profit that has been achieved. Incentives may be given either individually or on a competitive basis, so that participants' performance is compared.

Subjects control the system by using the function keys as indicated in the bottom line of Figure 2. Pressing one of the keys interrupts the experimental run. When the F2 key is chosen, a table is shown (Figure 4) from which items and quantities to order can be selected.

Pressing the F3 key displays the last eight transactions for a specific item, chosen by the subject. The display is in the log book form shown in Figure 5. Each line corresponds to a single transaction, showing the date, the quantity entering or exiting the system, or the quantity ordered at that date, and the inventory balance. The display remains on the screen until any key is pressed.

The last function key is the F4 key. Pressing it leads to the display of the demand graph for the specified item (see Figure 3). The graph shows the daily demand from the beginning of the experiment as a vertical bar chart, where each bar represents a single day's demand. Hence, bars become narrower as the experiment progresses.

The system's activities are recorded in detail in a results file, which contains two types of information: (1) general information regarding the experimental run, including the values of the selected parameters and the final financial information (i.e., net profit, sales revenues, and costs); and (2) the log books for all items, each beginning with the general parameter values for the item, followed by a detailed table where all transactions concerning the item are recorded.

The data allow the study of a wide range of applied and theoretical issues. The average order quantities and scheduling may be extracted from the results file and compared with the formal values. For example, it is possible to assess the differential sensitivity to various parameters, or to reconstruct a learning process over time, showing improvements in subjects' performance.

\section{AN ILLUSTRATIVE EXPERIMENT}

To test the system and demonstrate its use, a pilot experiment was conducted. We deliberately chose the simplest possible configuration of parameters and the simplest inventory management model described above.

Essentially we attempted to test how intuitive decisions are affected by three variables that are known to have the primary effects on the formal models of inventory management (the demand, the ordering cost, and

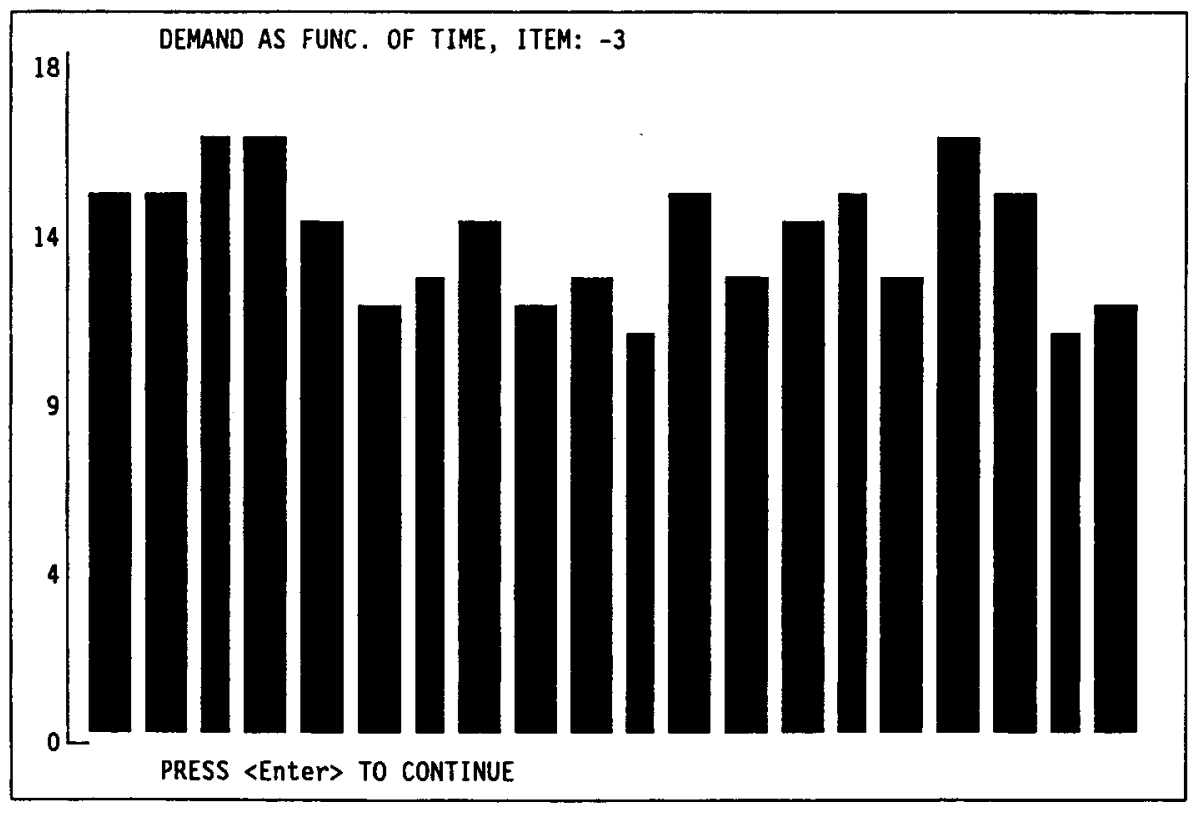

Figure 3. The item's demand chart. 


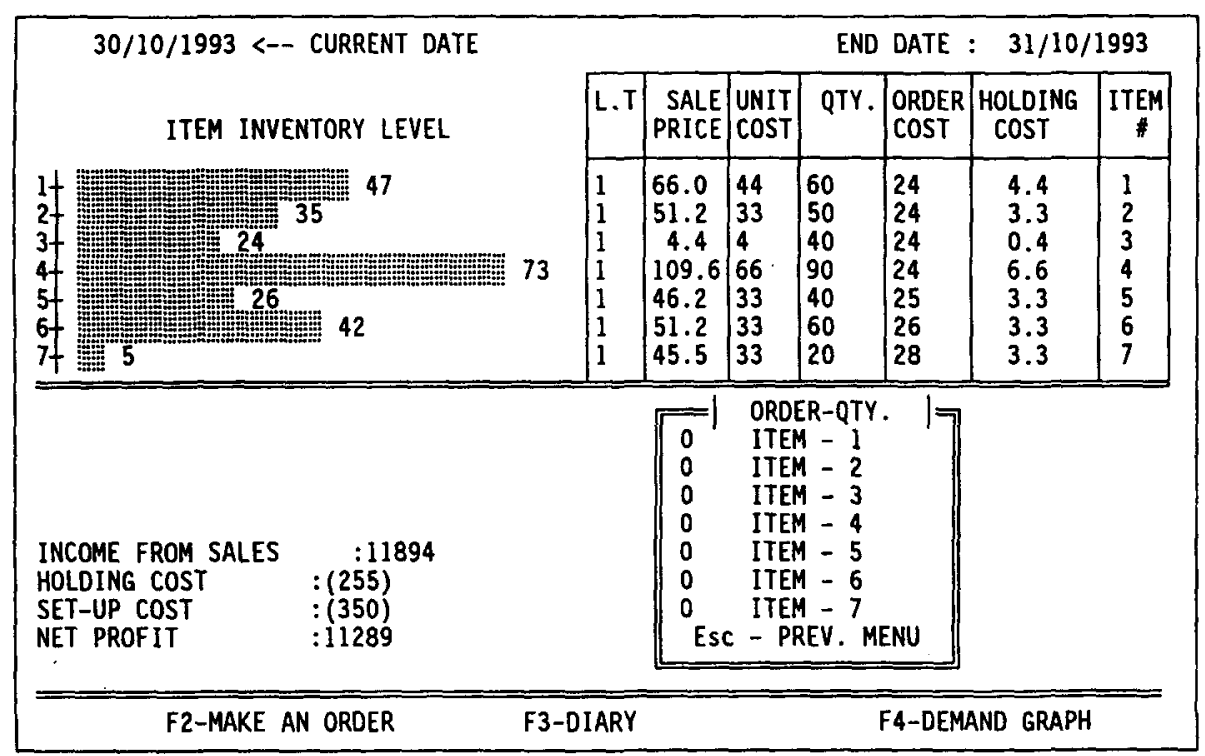

Figure 4. The ordering screen.

the inventory holding cost). Therefore a basic set of parameters was chosen, and this set of parameters was manipulated by keeping two of the parameters constant and changing the value of the third. Values were changed by diminishing each variable two steps from the basic value and by increasing it two steps from the basic value.

\section{Method}

Subjects. Twelve undergraduate students from various faculties volunteered to participate in this experiment.

Apparatus. The IIMS as outlined in the previous section served for running the experiment. The settings for this experiment were the simplest possible in the program:

1. The inventory system held only a single item.

2. The demand function was constant with no fluctuations. Therefore we also decided not to show subjects the demand curve.
3. Lead time was 1 day.

For this system we chose three basic values for the three parameters of interest, the demand ( $D=15)$, the ordering cost $(A=30)$, and the inventory holding cost $(h=2)$. For each of the three variables, four additional values were chosen in such a way that two values were smaller than the basic value and two were larger. Hence, for the demand, the values were $5,10,15,20$, and 25 ; for the ordering cost, the values were $10,20,30,40$, and 50 ; and for the inventory holding cost, the values were $0.5,1,2,3$, and 4 .

Since there were four variations from the basic combination of values for each of the three parameters, there were 12 different sets of variables, in addition to the basic set. These 13 sets allow functions to be drawn for each of the variables, where each function has 5 data points. Since the formal solutions for each of the five points is known, intuitive behavior can easily be compared with them.

Procedure. Subjects participated in the experiment individually. They received a brief explanation of the experimental system and

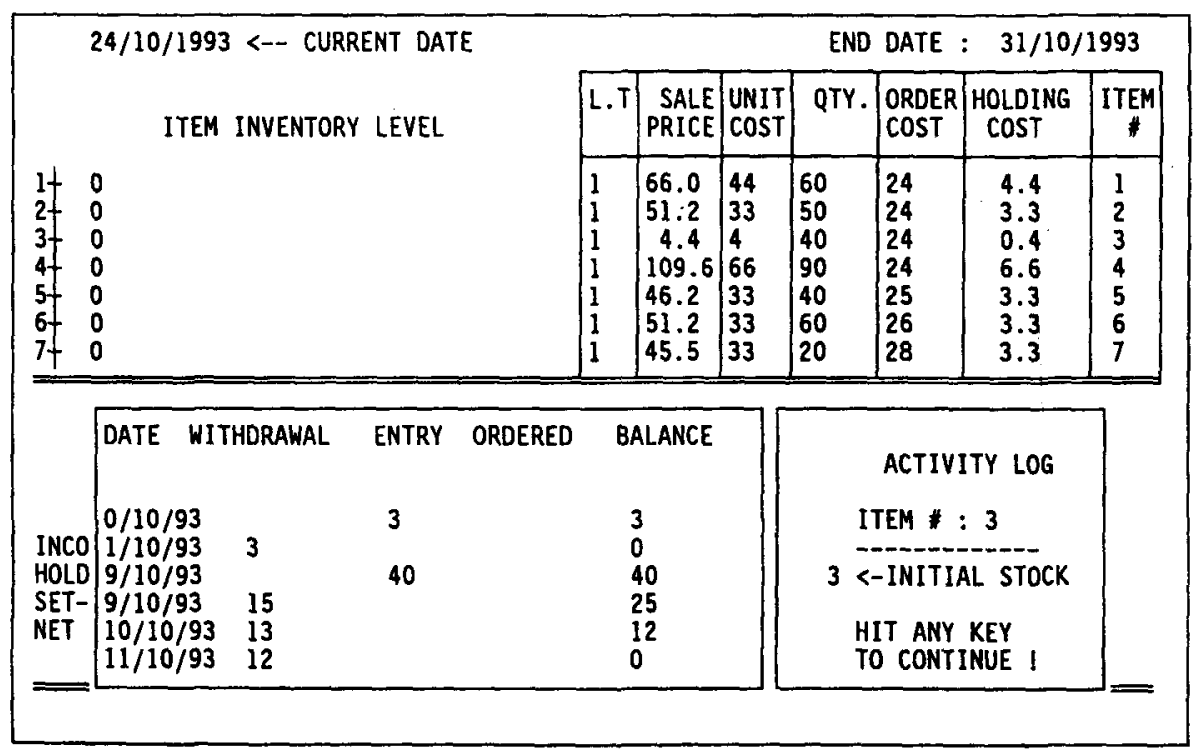

Figure 5. The item's log book. 
were shown how to read the various pieces of information from the screen and how to place an order. Then they received one practice trial (with parameters that differed from those used in the experiment itself). They were also told that they should try to maximize their profits, since the participant with the highest profit among all participants of the experiment would receive a monetary reward of about $\$ 15$.

Then the experiment itself began. The subjects were required to manage the inventory for the 13 sets of parameters. The sets were ordered randomly for each subject. Each subject was required to handle each set for 2 min of running time, which was considered to be 30 days, with each day represented by $4 \mathrm{sec}$. Since the running of the simulation was frequently interrupted by subjects' placing orders, the actual time for running each set was usually between 3 and $4 \mathrm{~min}$, and about $\mathrm{lh}$ was required for completion of the experiment.

\section{Results and Discussion}

All inventory transactions, entries and withdrawals, were recorded in the system's $\log$ for each of the 12 participants and for each of the 13 trials. These 156 data sets were then analyzed in order to determine the effects of the demand rate, the ordering cost, and the holding cost on order quantity and timing. Recall the calculation of the optimal order quantity for the basic model that was used in the experiment:

$$
Q^{*}=\sqrt{2 A D / h} .
$$

Since the order quantity is fixed, the ordering periodthe time between subsequent orders, $T$-should also be fixed:

$$
T^{*}=Q^{*} / D=\sqrt{2 A / D h}
$$

Thus, the effects of the parameters of interest on the formal model are clear. Our analysis aimed at comparing the intuitive behavior with that implied by the formal model. The comparison is shown in Figure 6. Both the average order quantities, top draw, and the average length of the ordering periods, bottom draw, are plotted as functions of the demand rate, the holding cost, and the ordering cost, along with the theoretic curves. To clarify the presentation, the parameters, on the $x$-axis, were transformed so that the formal function would be linear. For
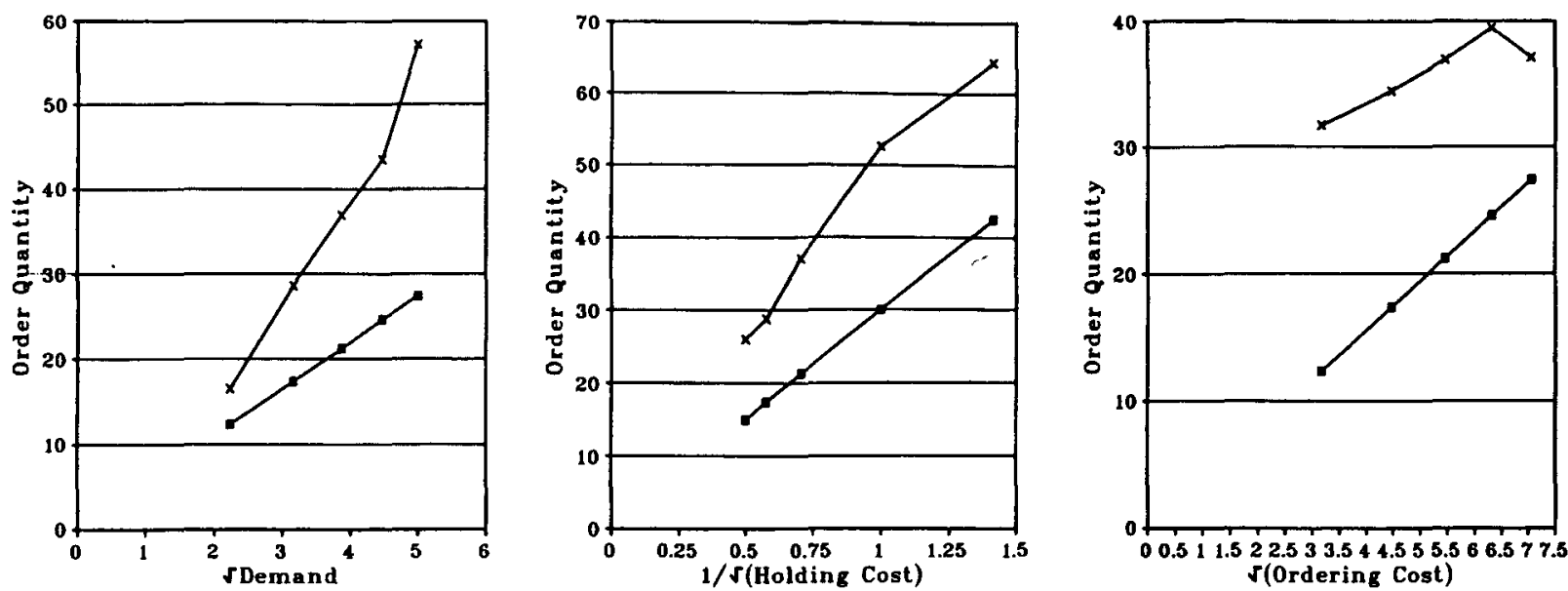

$X$ Experimental

- Theoretic

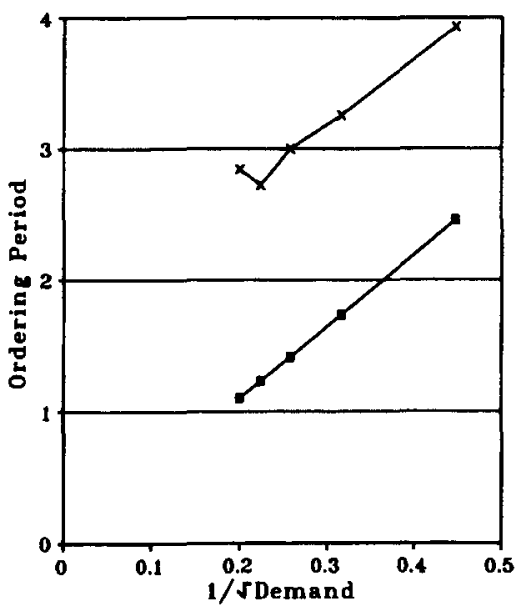

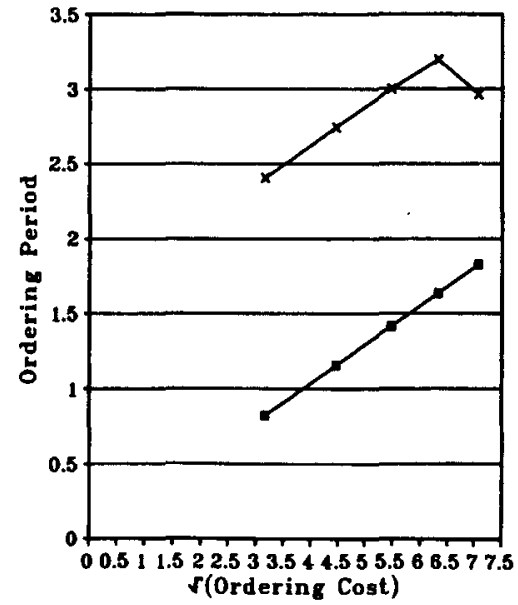

Figure 6. Experimentally observed and optimal order quantities and ordering periods. 
example, the optimal order quantity is a linear function of the square root of the demand rate and of the reciprocal of the square root of the holding cost.

Clearly, the quantities ordered by the participants are consistently larger than desired, and consequently, the ordering periods are longer (thus implying higher inventories and higher holding cost). Also, the general trends of the intuitive decisions are in the same directions as those suggested by the formal model, at least with regard to the demand rate and the holding cost. A question is suggested by the rates, the slopes of the curves. It seems that the intuitive decision maker overreacts in terms of the order quantity to changes in the demand rate and the holding cost, as indicated by the steeper slopes of the experimental curves. However, the experimental ordering period curves look parallel to the theoretic lines. Statistical tests confirm these results. The experimental slopes are steeper than the theoretical slopes for the quantity curves $[t(3)=3.4, p<.025$, for the quantity-holding cost curve, and $t(3)=6.65, p<$ .005 , for the quantity-demand curve], but there is no significant difference for the ordering period curves $[t(3)<$ 1 in both cases].

The effect of the ordering cost on both the order quantity and the ordering period is not clear and seems inconsistent. The slopes of both curves are shallow, relative to the other parameters, and the direction is changed. Statistically, the slope of the experimental quantity-ordering cost curve is lower than the theoretical slope $[t(3)=4.15$, $p<.025]$, but no significant difference appears for the ordering period curve.

In sum, the following behavioral patterns are apparent: the experimental ordering period curves are consistently higher but parallel to the theoretic lines; the experimental quantity curves have steeper slopes with respect to demand rate and holding cost and shallow slopes with respect to the ordering cost. This means that the intuitive decision maker correctly responds to changes in terms of time, the ordering period. The responses in terms of quantities are incorrect. The intuitive decision maker overreacts to changes in the demand rate and the holding cost and underreacts to changes in the ordering cost. It is noteworthy that quantities and time are dependent, $Q=D T$. Thus, the behavioral differences indicate inconsistency in intuitive decision making.

Finally, note that our conclusions are based on actual responses of human subjects to dynamic changes in the environment as opposed to the results of other possible methodologies, such as the use of questionnaires to study intuitive inventory decisions, which appear to have less ecological validity.

\section{SUMMARY}

The IIMS presents a novel approach to the study of intuitive decision making in an applied context. By simulating the operation of a complex system and by manipulating various parameters in this system, one can study intuitive decisions and determine their relation to the formal problem solutions. The system is highly versatile and allows integrative research on numerous issues in applied cognition to be conducted. It provides the opportunity to study the way in which people cope with complex systems that involve numerous interrelated variables. It is relevant both for understanding intuitive behavior in the domain of inventory management and at a more general level, where the case of inventory management serves as a tangible example for a complex system. In addition, the system may eventually be used as a demonstration tool in teaching and as a practical training tool for inventory managers.

\section{REFERENCES}

FUNKE, J. (1991). Solving complex problems: Exploration and control of complex systems. In R. J. Sternberg \& P. A. Frensch (Eds.), Complex problem solving: Principles and mechanisms (pp. 185-222). Hillsdale, NJ: Erlbaum.

FuRnHAM, A. (1988). Lay theories: Everyday understanding of problems in the social sciences. Oxford, U.K.: Pergamon Press.

Gentner, D., \& Stevens, A. L. (1983). Mental models. Hillsdale, NJ: Erlbaum.

Hax, A. C., \& Candea, D. (1984). Production and inventory management. Englewood Cliffs, NJ: Prentice-Hall.

Johnson, L. A., \& Montgomery, D. C. (1974). Operations research in production planning, scheduling, and inventory control. New York: Wiley.

Kahneman, D., SLovic, P., \& TVersky, A. (1982). Judgment under uncertainty: Heuristic and biases. New York: Cambridge University Press.

KLEITER, G. D. (1970). Trend control in a dynamic decision making task. Acta Psychologica, 34, 387-397.

MEYER, J., \& SHINAR, D. (1992). Estimating correlations from scatterplots. Human Factors, 34, 335-349.

Moray, N., Dessouky, M. I., Kijowski, B. A., \& Adapathya, R. (1991). Strategic behavior, workload, and performance in taskscheduling. Human Factors, 33, 607-629.

SANDERSON, P. M. (1989). The human planning and scheduling role in advanced manufacturing systems: An emerging human factors domain. Human Factors, 31, 635-666.

(Manuscript received November 18, 1993; revision accepted for publication August 30, 1994.) 